\title{
BMJ Open Association study of TREM2 polymorphism rs75932628 with leucoaraiosis or Parkinson's disease in the Han Chinese population
}

\author{
Zhiming Li, ${ }^{1}$ Li Zhong, ${ }^{2}$ Long Gu, ${ }^{1}$ Wenqing Huang, ${ }^{1}$ Xinzhen Shi, ${ }^{2}$ Xilin Zhang, ${ }^{2}$ \\ Xingkai An, ${ }^{3}$ Qing Lin, ${ }^{1,3}$ Chi-Meng Tzeng ${ }^{1}$
}

To cite: Li Z, Zhong L, Gu L, et al. Association study of TREM2 polymorphism rs75932628 with

leucoaraiosis or Parkinson's disease in the Han Chinese population. BMJ Open 2016;6:e009499. doi:10.1136/bmjopen-2015009499

- Prepublication history for this paper is available online. To view these files please visit the journal online (http://dx.doi.org/10.1136/ bmjopen-2015-009499).

ZL and LZ contributed equally.

Received 22 July 2015 Revised 18 November 2015 Accepted 19 November 2015

CrossMark

For numbered affiliations see end of article.

Correspondence to Professor Chi-Meng Tzeng; cmtzeng@xmu.edu.cn

\section{ABSTRACT}

Objectives: The previously reported functional mutation rs75932628-T (p.R47H) in the triggering receptor expressed on myeloid cells 2 (TREM2) is a genetic risk factor for Alzheimer's disease, Parkinson's disease (PD) and frontotemporal dementia, in European populations. This study aims to assess the genetic association of the variant rs75932628-T with PD and leucoaraiosis (LA) in a Han Chinese population.

Setting: This population-based study was conducted in China by Xiamen University and its affiliated hospital.

Participants: 308 patients with LA, 342 patients with PD and 198 healthy blood donors were recruited from the First Affiliated Hospital of Xiamen University.

Outcome measures: Genotyping was performed by molecular beacon real-time PCR and Sanger sequencing.

Results: None of our participants carried the rs75932628-T mutation.

Conclusions: Our results corroborate and extend previous findings, concluding that the variant rs75932628-T (p.R47H) in TREM2 is not a risk factor for LA or PD in the Han Chinese population.

\section{INTRODUCTION}

The triggering receptor expressed on myeloid cells 2 (TREM2) is a membrane receptor expressed on macrophages and microglia in the central nervous system. ${ }^{1}$ Functional studies have shown that TREM2 not only suppresses inflammation but also promotes the phagocytosis of apoptotic neurons through TREM2 ligation. ${ }^{2}$ Recently, several studies demonstrated that the non-synonymous mutation rs75932628-T, p.R47H, increases the risk of certain neurodegenerative diseases, including Alzheimer's disease (AD),$^{3-5}$ Parkinson's disease (PD) ${ }^{6} 7$ and frontotemporal dementia, ${ }^{4} 6$ in European populations. In addition,

\section{Strengths and limitations of this study}

- This study was the first Han Chinese populationbased study to evaluate the polymorphism rs75932628-T (p.R47H) in leucoaraiosis (LA), and to validate the previous results of rs75932628-T (p.R47H) in Parkinson's disease (PD) from the Caucasian population.

- The results suggested that the rs75932628-T ( $p$. $\mathrm{R} 47 \mathrm{H}$ ) variation in triggering receptor expressed on myeloid cells 2 gene may not be a genetic risk factor for LA and PD in the Han Chinese population.

- Sanger sequencing was used to verify the results of the beacon real-time PCR.

- The population size may be limited and future studies with larger population sizes are needed to test our results.

homozygous loss-of-function mutations in TREM2 are genetic causes of patients with Nasu-Hakola disease, also known as polycystic lipomembranous osteodysplasia with sclerosing leucoencephalopathy (PLOSL). Leucoencephalopathy, such as PLOSL, is characterised by diffuse low-density changes in the cerebral white matter, showing low density by CT and hyperintensity by T2-weighted or fluid attenuated inversion recovery (FLAIR). Neurologists often encounter this imaging appearance, but the mechanisms of leucoaraiosis (LA) remain unknown. The above findings led us to hypothesise that this variation might confer susceptibility to LA. Therefore, we analysed whether rs75932628-T in TREM2 would increase the risk of LA or PD in a large northern Han Chinese population.

\section{EXPERIMENTAL PROCEDURES}

Subjects

A total of 342 patients with PD, 308 patients with LA and 198 age-matched and gender- 
matched healthy controls were enrolled from the First Affiliated Hospital of Xiamen University. The demographic characteristics of the study population are displayed in table 1. All participants underwent brain imaging. Patients with LA and PD were diagnosed independently by two neurologists. Patients with LA were recruited according to conventional MRI on a 1.5-T system, including transverse T2-weighted/T1-weighted and FLAIR sequences, and sagittal T1 with $5 \mathrm{~mm}$ thick slices. LA was determined as a bilateral and symmetrical area in the periventricular and centrum semiovale, showing a white matter lesion with hyperintensities in T2-weighted and FLAIR images. PD diagnostic criteria were based on the UK PD Brain Bank. The severity of PD was assessed by the Unified Parkinson's Disease Rating Scale. Patients with PD with white matter lesions were excluded. The control group also underwent neuroimaging examination to exclude LA. Informed and written consent was obtained from patients before blood collection.

\section{DNA extraction and molecular beacon real-time PCR}

Genomic DNA of all participants was isolated from peripheral whole blood using a MagCore Genomic DNA Whole Blood Kit and HF-16 extractor (RBC Bioscience, Taiwan), as previously published. ${ }^{9}$ Genotyping of rs75932628-T (p.R47H) was conducted by molecular beacon real-time PCR using an ABI 7500 Fast Real-Time PCR System (Applied Biosystems, Foster City, California, USA) and then confirmed by Sanger sequencing using an ABI 3730XL automatic sequencer (Applied Biosystems, Foster City, California, USA). Sequences of primers and molecular beacons are given in table 2 . The amplification procedure consisted of $95^{\circ} \mathrm{C}$ for $20 \mathrm{~s}$ followed by 40 cycles of $95^{\circ} \mathrm{C}$ for $3 \mathrm{~s}, 54^{\circ} \mathrm{C}$ for $30 \mathrm{~s}$ and $72^{\circ}$ $\mathrm{C}$ for $10 \mathrm{~s}$. The fluorescence spectra of the molecular beacons were measured during the annealing step of the PCR cycle. The reference controls were two custommade plasmids (Sangon Biotech, Shanghai, China).

\section{Statistical analysis}

Fisher's exact test was used to compare the distribution of genotype frequency of the rs75932628-T in the casecontrol study. All analysis was conducted using the

\begin{tabular}{|c|c|}
\hline $\begin{array}{l}\text { Primers and } \\
\text { molecular } \\
\text { beacons }\end{array}$ & Sequence $\left(5^{\prime}-3^{\prime}\right)$ \\
\hline \multicolumn{2}{|l|}{ Primers } \\
\hline Sense & GTGTCTTGCCCСTATGACTCCA \\
\hline Antisense & GTGCTCCCАTTCCАССТССТ \\
\hline \multicolumn{2}{|c|}{ Molecular beacons } \\
\hline \multirow[t]{3}{*}{ WT } & FAM-5'-CGGTCA \\
\hline & ACTGGGGGAGGCGCAAGG \\
\hline & TGACCG-3'-BHQ1 \\
\hline \multirow[t]{3}{*}{ Mutation } & HEX-5'-CGCGTA \\
\hline & TGGGGGAGGCACAAGGC \\
\hline & TACGCG-3'-BHQ̄1 \\
\hline
\end{tabular}

statistical software SPSS V.20.0 for Windows (IBM SPSS Inc, Chicago, Illinois, USA).

\section{RESULTS}

Among a total of 848 individuals, we did not find any allelic variant rs75932628-T, using molecular beacon real-time PCR. Furthermore, this result was also validated by Sanger sequencing, suggesting that the previously and notably reported rs75932628-T in European populations exhibits a significantly lower incidence in the northern Han Chinese population. PCR amplification curves (A) and Sanger sequencing (B) are displayed in figure $1 \mathrm{~A}, \mathrm{~B}$, respectively. Our data corroborate and extend previous findings and conclude that rs75932628-T is not a genetic risk factor for PD in the Chinese population. ${ }^{10}$ More importantly, we first screened rs75932628-T among patients with LA, thus suggesting that rs75932628-T may not be a risk factor for LA in the Chinese population.

\section{DISCUSSION}

It has been revealed that neuroinflammation may contribute to the pathogenesis of ageing disorders, such as PD and LA. Given that TREM2 plays an important role in the immune response, and considering the heterogeneous phenotype observed in carriers of TREM2 mutations in European populations, we hypothesised that the

Table 1 Demographic characteristics of the study population

\begin{tabular}{llllll}
\hline Characteristics & $\begin{array}{l}\text { Patients with LA } \\
(\mathbf{n = 3 0 8 )}\end{array}$ & $\begin{array}{l}\text { Patients with PD } \\
(\mathbf{n}=\mathbf{3 4 2})\end{array}$ & $\begin{array}{l}\text { Control subjects } \\
(\mathbf{n}=198)\end{array}$ & $\begin{array}{l}\mathbf{p}^{\text {LA }} \\
\text { Value }\end{array}$ & $\begin{array}{l}\mathbf{p}^{\text {PD }} \\
\text { Value }\end{array}$ \\
\hline $\begin{array}{l}\text { Age }(\text { mean } \pm S D, \text { year) } \\
\text { Gender, } n(\%) \dagger\end{array}$ & $62.83 \pm 9.89$ & $61.34 \pm 10.29$ & $61.59 \pm 8.27$ & 0.113 & 0.746 \\
$\quad \begin{array}{l}\text { Male } \\
\text { Female }\end{array}$ & $142(46.1)$ & $162(47.4)$ & $98(49.5)$ & 0.456 & 0.634 \\
\hline
\end{tabular}

*Student $t$ test was used to compare mean age between patients with LA or PD, and control subjects.

†Fisher's exact test was used to assess the difference in gender distribution between patients with LA or PD, and control subjects.

LA, leucoaraiosis; PD, Parkinson's disease. 
A

Amplification Plot

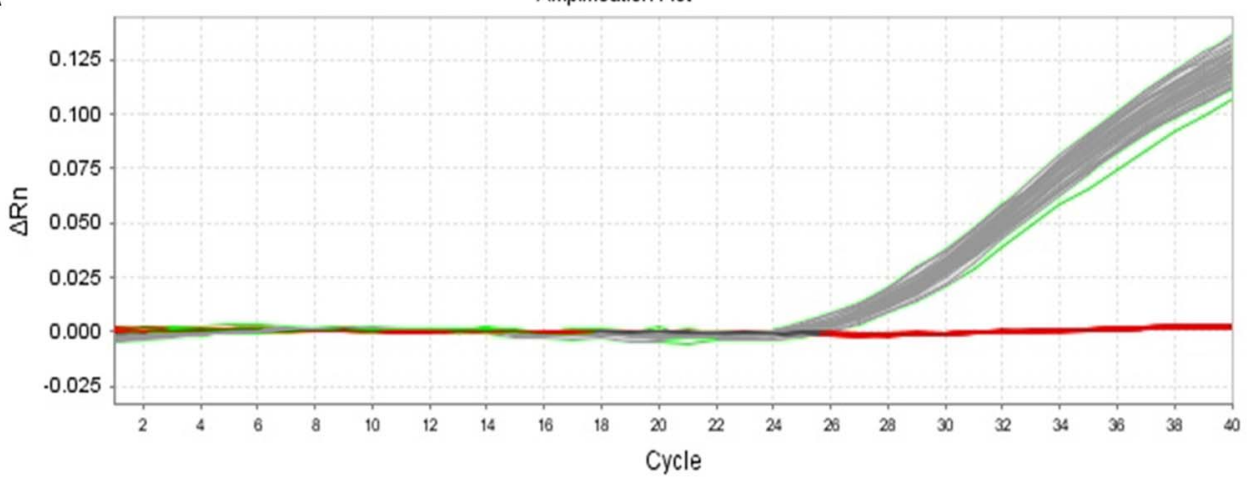

TREM2-MUTATION TREM2-WT I SAMPLES

B

TREM2-WT : rs75932628

$\begin{array}{cccccc}150 & 160 & 170 & 180 & 190 & 200\end{array}$

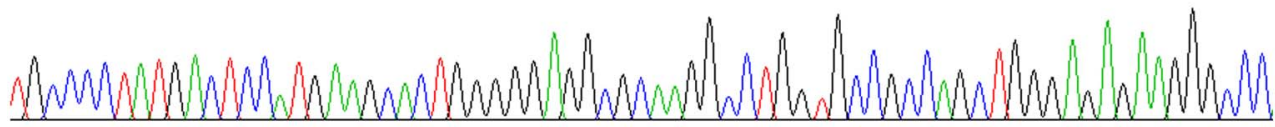

TREM2-MUTATION : rs75932628

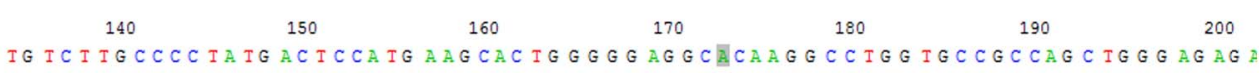

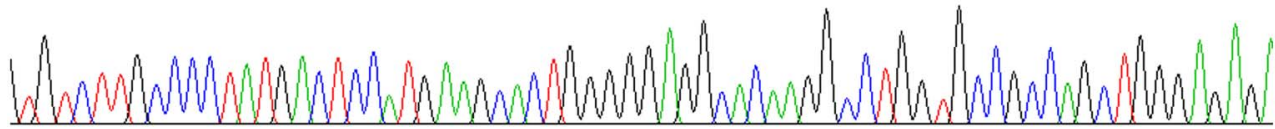

Figure 1 PCR amplification curve (A) and Sanger sequencing (B). (A) The molecular beacon specific for the major (G) allele (wild type, WT) is FAM labelled, and the molecular beacon specific for the minor (A) allele (mutation) is HEX labelled. The fluorescence spectra of the molecular beacons were measured during the annealing step of the PCR cycle. (B) The dark-coloured region of the sequence indicates the rs75932628-T (p.R47H) site in both, the wild and the mutant types.

non-synonymous variant rs75932628-T in TREM2 might be a risk factor for PD and LA in the Han Chinese population. In this study, we genotyped this variant in a number of well-characterised disease patients with PD and LA, as well as in a series of controls. However, neither the heterozygous nor the homozygous mutation, rs75932628-T, was carried by the participants, indicating that the minor allele frequency (MAF) for rs75932628- $\mathrm{T}$ is extremely low in our population. Our findings were consistent with those from previous reports. Recently, Feng and colleagues reported that the MAF of this rare variant in a Han Chinese population was only $0.06 \%$, $0.2 \%(1 / 476)$ of patients with PD and was $0(0 / 432)$ in the controls. ${ }^{10}$ Moreover, in patients with $\mathrm{AD}$ in a Han Chinese population, rs75932628-T was not found by directly sequencing exon 2 of TREM $2 .{ }^{11}$

Since 2013, two independent groups have identified rs75932628-T (causing p.R47H substitution) as being strongly associated with late-onset $\mathrm{AD}$ in cohorts from
Iceland, Germany, the Netherlands, Norway and the USA. $^{3}{ }^{12}$ This finding was successfully replicated in French and Spanish populations. ${ }^{11} 1314$ Meta-analysis of 11 previously reported $\mathrm{AD}$ cohorts validated the correlation of rs75932628-T with $\mathrm{AD}\left(\mathrm{p}=2.93 \times 10^{-17}\right)$ in populations of European descent. ${ }^{4}$ Notably, neuroimaging showed that many patients had LA, also referred to as white matter lesions, which are prevalently observed in PLOSL, AD and PD. LA is considered to contribute to dementia in $\mathrm{AD}$, and emerging but inconclusive evidence has shown similar effects in PD. Some researchers have suggested that LA may exacerbate or contribute to some motor and cognitive deficits related to PD. ${ }^{15}$ To date, no study has investigated the association between rs75932628-T in TREM2, and LA, in any population. This study is the first to investigate the association of TREM2 and LA in a southern Han Chinese population. In addition, our result is consistent with Feng's report that rs75932628-T is not a risk factor for PD in the 
southern Han Chinese population. Similarly, no significant association with late-onset $\mathrm{AD}$ and rs75932628-T was observed in a large Japanese population. ${ }^{16}$

In summary, our results, combined with Yu's and Feng's studies consisting of more than 4000 participants, indicate that the variant rs75932628-T (p.R47H) of TREM2 is unlikely to contribute to the pathogenesis of $\mathrm{LA}, \mathrm{PD}$ and $\mathrm{AD}$, or susceptibility to these diseases, in the Chinese population. This result, which is distinct from those in the original reports, might arise mainly from ethnic variation between Chinese populations and those of European descent. However, future studies should be performed using larger sample sizes, and including gene-gene and gene-environmental interactions.

\section{Author affiliations}

${ }^{1}$ Translational Medicine Research Center, School of Pharmaceutical Sciences, Xiamen University, Xiamen, Fujian, China

${ }^{2}$ Fujian Provincial Key Laboratory of Neurodegenerative Disease and Aging Research, Institute of Neuroscience, College of Medicine, Xiamen University, Xiamen, Fujian, China

${ }^{3}$ Department of Neurology, The First Affiliated Hospital of Xiamen University, Xiamen, Fujian, China

Acknowledgements The authors would like to thank the language editing service of the Nature Publishing Group.

Contributors XA and QL collected the blood samples from patients with PD and $L A$, and from controls, extracted genomic DNA and searched the relevant literature. ZL and LZ performed PCR analysis, and built positive and negative plasmids. WH and XS designed the primers for PCR and performed sequence alignment. XZ and LG participated in statistical analysis and the production of tables. ZL and C-MT designed and guided all the experiments, and drafted and revised the manuscript.

Funding This study was funded by the National Natural Science Fund (81400912) and the Science and Technology Project of Fujian Province (number 2012D062), China.

Competing interests None declared.

Patient consent Obtained.

Ethics approval The study was approved by the Ethics Committee of Xiamen First Hospital.

Provenance and peer review Not commissioned; externally peer reviewed.

Data sharing statement No additional data are available.
Open Access This is an Open Access article distributed in accordance with the Creative Commons Attribution Non Commercial (CC BY-NC 4.0) license, which permits others to distribute, remix, adapt, build upon this work noncommercially, and license their derivative works on different terms, provided the original work is properly cited and the use is non-commercial. See: http:// creativecommons.org/licenses/by-nc/4.0/

\section{REFERENCES}

1. Colonna M. TREMs in the immune system and beyond. Nat Rev Immunol 2003;3:445-53.

2. Hsieh CL, Koike M, Spusta SC, et al. A role for TREM2 ligands in the phagocytosis of apoptotic neuronal cells by microglia. $J$ Neurochem 2009;109:1144-56.

3. Guerreiro R, Wojtas A, Bras J, et al. TREM2 variants in Alzheimer's disease. N Engl J Med 2013;368:117-27.

4. Cuyvers E, Bettens K, Philtjens S, et al. BELNEU consortium. Investigating the role of rare heterozygous TREM2 variants in Alzheimer's disease and frontotemporal dementia. Neurobiol Aging 2014;35:726.e11-19.

5. Rosenthal SL, Bamne MN, Wang X, et al. More evidence for association of a rare TREM2 mutation (R47H) with Alzheimer's disease risk. Neurobiol Aging 2015;36:2443.e21-6.

6. Rayaprolu S, Mullen B, Baker M, et al. TREM2 in neurodegeneration: evidence for association of the p.R47H variant with frontotemporal dementia and Parkinson's disease. $\mathrm{Mol}$ Neurodegener 2013;8:19.

7. Jonsson T, Stefansson K. TREM2 and neurodegenerative disease. N Engl J Med 2013;369:1568-9.

8. Klünemann $\mathrm{HH}$, Ridha $\mathrm{BH}$, Magy L, et al. The genetic causes of basal ganglia calcification, dementia, and bone cysts: DAP12 and TREM2. Neurology 2005;64:1502-7.

9. Ma Q, An X, Li Z, et al. P268S in NOD2 associates with susceptibility to Parkinson's disease in Chinese population. Behav Brain Funct 2013;9:19.

10. Feng SJ, Nie K, Gan R, et al. Triggering receptor expressed on myeloid cells 2 variants are rare in Parkinson's disease in a Han Chinese cohort. Neurobiol Aging 2014;35:1780.e11-12.

11. $\mathrm{Yu}$ JT, Jiang $\mathrm{T}$, Wang $\mathrm{YL}$, et al. Triggering receptor expressed on myeloid cells 2 variant is rare in late-onset Alzheimer's disease in Han Chinese individuals. Neurobiol Aging 2014;35:937.e1-3.

12. Jonsson $\mathrm{T}$, Stefansson $\mathrm{H}$, Steinberg $\mathrm{S}$, et al. Variant of TREM associated with the risk of Alzheimer's disease. $N$ Engl J Med 2013;368:107-16

13. Pottier C, Wallon D, Rousseau S, et al. TREM2 R47H variant as a risk factor for early-onset Alzheimer's disease. J Alzheimers Dis 2013;35:45-9.

14. Benitez BA, Cooper B, Pastor $\mathrm{P}$, et al. TREM2 is associated with the risk of Alzheimer's disease in Spanish population. Neurobiol Aging 2013;34:1711.e15-17.

15. Bohnen NI, Albin RL. White matter lesions in Parkinson disease. Nat Rev Neurol 2011;7:229-36.

16. Miyashita A, Wen Y, Kitamura N, et al. Lack of genetic association between TREM2 and late-onset Alzheimer's disease in a Japanese population. J Alzheimers Dis 2014;41:1031-8. 